Letter

\title{
Intrinsic Interference Use for FBMC-IOTA Systems
}

\author{
Lei Wen ${ }^{1,2}$, Razieh Razavi ${ }^{2}$ and Jing Lei ${ }^{1, *}$ \\ 1 College of Electronics Science, National University of Defense Technology, Changsha 410000, Hunan, China \\ 2 5GIC, University of Surrey, Guildford GU2 7XH, UK \\ * Correspondence: leijing@nudt.edu.cn
}

Received: 4 June 2019; Accepted: 2 August 2019; Published: 7 August 2019

\begin{abstract}
In this paper, the intrinsic interference of filter bank-based multicarrier systems (FBMC) systems with isotropic orthogonal transfer algorithm (IOTA) pulse-shaping is analyzed and used. Such intrinsic interference is treated as a parity symbol, and an iterative soft-in-soft-out (SISO) detector, which is based on message-passing algorithm (MPA), is proposed to exploit the useful information of the intrinsic interference. The performance of the intrinsic interference user (IIU) is investigated.
\end{abstract}

Keywords: filter bank multicarrier; isotropic orthogonal transfer algorithm; intrinsic interference

\section{Introduction}

To mitigate the effect of multipath fading, a cyclic prefix $(\mathrm{CP})$ must be inserted to orthogonal frequency division multiplexing (OFDM) systems, resulting in a reduced spectral efficiency and an increased power consumption. By partitioning the wideband channel into a large number of parallel narrow band sub-channels, the task of high-data-rate transmission over a frequency selective channel has been transformed into number of parallel low-data-rate transmissions which need equalization techniques. Some improvements for OFDM have been reported to combat frequency dispersion sensitivity by exploiting ICI self-cancellation methods [1,2] or to explore space and time diversity in dispersive channels through fractional sampling [3,4]. And numerous research efforts have been spent on PAPR reduction techniques. The usage of $\mathrm{CP}$, however, is retained to combat ISI in such techniques which aim to enhance OFDM [5,6]. In [7-10], with reference to the problem of joint equalization and narrowband interference (NBI) suppression in OFDM systems, synthesis and analysis of both unconstrained and constrained optimum equalizers are carried out, based on the maximum signal-to-noise-plus-interference (SINR) criterion. An alternative approach is to use pulse-shaping filters. With offset modulations, for example, OFDM/offset quadrature amplitude modulation (OQAM), the orthogonality can be maintained with proper pulse-shaping [11,12]. OFDM with offset QAM (OFDM/OQAM) which transmits real symbols with double lattice density has shown some advantages over OFDM, but faces difficulties of channel estimation and equalization [13]. It is shown that OFDM/OQAM with isotropic orthogonal transfer algorithm (IOTA) pulse-shaping, which is called FBMC-IOTA, has optimal localization property [14]. FBMC-IOTA has been studied in [15] and was shown to outperform conventional OFDM over different types of wireless propagation channels.

Despite all the advantages, the currently developed FBMC-IOTA system is not yet fully used to its best achievable performance. In the FBMC-IOTA receiver, there is intrinsic interference which contains rich information, and its effect on the spectral efficiency has been analyzed from information theoretical perspective in [16]. However, to the best of our knowledge, there does not exist any method to exploit the extra information offered by the intrinsic interference in the symbol detection process. This motivates us to develop an algorithm to use the intrinsic interference to materialize the performance gain predicted by the information theoretical study. In this contribution we show that the 
intrinsic interference can be determined by multiplying the neighboring symbols with a weighting matrix, the elements of which can be calculated using the ambiguity function of the pulse shape filter. This is essentially the principle of block coding and the intrinsic interference serves as non-binary parity symbol. Using this property and message-passing algorithm (MPA) [17], we propose a way which is termed as intrinsic interference user (IIU) to improve the system performance. Although FBMC-IOTA can mitigate ISI, if the receiver has estimated the channel well enough, then ISI can be further cleaned up using MPA on the demodulated reception. This translates to roughly $1 \mathrm{~dB}$ bit error rate (BER) improvement across SNR's for a particular channel model.

The rest of the paper is outlined as follows. Section 1 presents the maximum a posteriori (MAP) detection on weight matrix of FBMC-IOTA. In Section 2, MPA on the intrinsic interference of FBMC-IOTA is presented. Section 3 presents simulation results. Conclusions are drawn in Section 4.

\section{MAP Detection on Weight Matrix}

Consider a FBMC-IOTA system with $N$ subcarriers and $L$ symbols, the transmitted signal can be written as

$$
s(t)=\sum_{n=0}^{L-1} \sum_{m=0}^{N-1} a_{m, n} g_{m, n}(t)
$$

where $a_{m, n}$ and $g_{m, n}(t)$ represent the symbol modulated by the $m$ th subcarrier at the $n$th symbol time and the synthesis basis obtained by the IOTA function $g(t)$, respectively.

After passing by a doubly dispersive channel, the demodulator output can be expressed as [15]

$$
\begin{aligned}
\hat{a}_{k, l} & =n_{k, l}+a_{k, l} H_{k, l} \\
& +\underbrace{\sum_{p, q \neq 0} a_{k+p, l+q} j^{p+q+p(q+2 l)} A_{g}^{*}\left(q \tau_{0}, p v_{0}\right)}_{I_{k, l}} H_{k, l}
\end{aligned}
$$

where $H_{k, l}$ is the channel coefficient at the $l$ th symbol and the $k$ th subcarrier frequency, $A_{g}(\tau, v)$ is the auto-ambiguity function of $g(t), n_{k, l}$ is the channel noise, $I_{k, l}$ is the intrinsic interference and can be written in the form of $I_{k, l}=j a_{k, l}^{(i)}$. Such intrinsic interference contains rich inherent information that has never been used. In fact, the intrinsic interference can be determined by multiplying the neighboring symbols with a weighting matrix, the elements of which can be calculated using the ambiguity function of the employed pulse shape filter [16]. The matrix that presents the weights corresponding to each neighboring symbol is shown below

$$
\begin{aligned}
W_{l_{\text {even }}}= & {\left[\begin{array}{ccccccc}
0 & 0 & -\eta & 0 & \eta & 0 & 0 \\
-\lambda & -\zeta & -\gamma & -\delta & -\gamma & -\zeta & -\lambda \\
-\theta & 0 & -\beta & 1 & \beta & 0 & \theta \\
-\lambda & \zeta & -\gamma & \delta & -\gamma & \zeta & -\lambda \\
0 & 0 & -\eta & 0 & \eta & 0 & 0
\end{array}\right] } \\
W_{l_{\text {odd }}} & =\left[\begin{array}{ccccccc}
0 & 0 & -\eta & 0 & \eta & 0 & 0 \\
\lambda & \zeta & \gamma & \delta & \gamma & \zeta & \lambda \\
-\theta & 0 & -\beta & 1 & \beta & 0 & \theta \\
\lambda & -\zeta & \gamma & -\delta & \gamma & -\zeta & \lambda \\
0 & 0 & -\eta & 0 & \eta & 0 & 0
\end{array}\right]
\end{aligned}
$$

where $l_{\text {even }}$ and $l_{\text {odd }}$ respectively corresponds to the weight for real and imaginary symbols, and $\delta=0.2486, \beta=0.5756, \gamma=0.1898, \eta=0.0021, \theta=0.0956, \lambda=0.0473, \zeta=0.0991$. 
For each transmitted information symbol $a_{k, l}$, its associated intrinsic interference $a_{k, l}^{(i)}$ is determined by neighboring symbols and can be calculated as in (2) using the above weighting matrices. We find that such principle is essentially the same as block coding where each symbol $a_{k, l}$ is associated with some non-binary parity symbols $P_{k, l}=a_{k, l}^{(i)}$. In the following we will focus on the real branch of FBMC-IOTA receiver, and the analysis can be applied to the imaginary branch straightforwardly.

Given $p=\left[P_{k, l}: k \in[0, \ldots, N-1], l \in[0, \ldots, L-1]\right]$ as the vector of observed parity symbols at real branch, the MAP detection will estimate $\hat{a}$ that maximizes the joint a posteriori probability mass function (PMF) of the transmitted symbol vector $\boldsymbol{a}=\left[a_{m, n}: m \in[0, \ldots, N-1], n \in[0, \ldots, L-1]\right.$, i.e.,

$$
\hat{\boldsymbol{a}}=\arg \max _{\boldsymbol{a}} p(\boldsymbol{a} \mid \boldsymbol{p}) .
$$

The MAP detector can be implemented by two approaches: individual and joint optimum detection $[18,19]$. The joint approach maximizes the joint a posteriori PMF of the transmitted symbol vector as shown above, while the individual optimum MAP detection maximizes the a posteriori PMF, $p\left(a_{k, l} \mid p\right)$, of each individual symbol. Let $\mathbb{X}$ be the constellation alphabet, from which $a_{m, n}$ will take its value, the estimation of $a_{k, l}$ with individual MAP detection can be written as

$$
\hat{a}_{k, l}=\arg \max _{a_{m, n} \in \mathbb{X}} p\left(a_{m, n} \mid \boldsymbol{p}\right)
$$

A posteriori PMF for $a_{k, l}$ can be found by calculating the marginal of the joint a posteriori PMF, thus (6) can be written as

$$
\hat{a}_{k, l}=\arg \max _{a_{m, n} \in \mathbb{X}} \sum_{\boldsymbol{a} \in \mathbb{X}^{N, L}} p(\boldsymbol{a} \mid \boldsymbol{p})
$$

Let $\operatorname{Pr}(a)$ be the priori probability of symbol $a$. According to Bayes' rule, we have

$$
p(\boldsymbol{a} \mid \boldsymbol{p}) \propto p(\boldsymbol{p} \mid \boldsymbol{a}) \operatorname{Pr}(\boldsymbol{a})
$$

where $\operatorname{Pr}(\boldsymbol{a})$ is the joint a priori PMF of all symbols assuming that they are independent to each other. Therefore, the estimation function can be modified to

$$
\hat{a}_{k, l}=\arg \max _{a_{m, n} \in \mathbb{X}} \sum_{\boldsymbol{a} \in \mathbb{X}^{N \times L}} p(\boldsymbol{p} \mid \boldsymbol{a}) \prod_{a \in \boldsymbol{a}} \operatorname{Pr}(a)
$$

As can be seen from (9) this computation includes a marginalization process which is NP-hard [20]. Furthermore, with the assumption that the noise vector is identically and independently distributed (i.i.d.) and is uncorrelated with the transmitted symbols, we can factorize

$$
p(\boldsymbol{p} \mid \boldsymbol{a})=\prod_{k^{\prime}, l^{\prime}} p\left(P_{k^{\prime}, l^{\prime}} \mid \boldsymbol{a}\right)
$$

Please note that $P_{k, l}$ is the parity symbol related to the symbol to be detected. Therefore, in this equation a general form of $P_{k^{\prime}, l^{\prime}}$ is applied. Since a limited number of symbols interfere on each parity symbol, the calculation of this function can be simplified as

$$
p(\boldsymbol{p} \mid \boldsymbol{a})=\prod_{k^{\prime}, l^{\prime}} p\left(P_{k^{\prime}, l^{\prime}} \mid \boldsymbol{a}^{\left[k^{\prime}, l^{\prime}\right]}\right)
$$

where $\boldsymbol{a}^{\left[k^{\prime}, l^{\prime}\right]}$ is the vector of symbols that interfere on the parity symbol with indices $k^{\prime}, l^{\prime}$. Substituting (11) into (9) yields

$$
\hat{a}_{k, l}=\arg \max _{a_{m, n} \in \mathbb{X}} \sum_{\boldsymbol{a} \in \mathbb{X}^{N \times L}} \operatorname{Pr}(\boldsymbol{a}) \prod_{k^{\prime}, l^{\prime}} p\left(P_{k^{\prime}, l^{\prime}} \mid \boldsymbol{a}^{\left[k^{\prime}, l^{\prime}\right]}\right)
$$


From (12), we can see that the FBMC-IOTA structure allows us to translate the MAP detection problem into the marginalize product of functions (MPF) problem. The local observation at parity symbol $P_{k^{\prime}, l^{\prime}}$ is given by

$$
p\left(P_{k^{\prime}, l^{\prime}} \mid \boldsymbol{a}^{\left[k^{\prime}, l^{\prime}\right]}\right)=\frac{1}{\sqrt{2 \pi} \sigma} \exp \left(\frac{1}{2 \pi \sigma^{2}}\left\|P_{k^{\prime}, l^{\prime}}-\boldsymbol{a}^{\left[k^{\prime}, l^{\prime}\right]} \boldsymbol{s}^{T}\right\|^{2}\right)
$$

where $s$ is the vector of calculated weights according to (3) and (4).

\section{MPA on Intrinsic Interference}

Although the joint MAP detection problem has been translated into an MPF problem as shown in (12) which is much simpler to resolve, the brute-force solution for (11) requires exponential complexity. In order to reduce the complexity, we propose a novel iterative symbol detector IIU based on the generic MPA [21], where MPA is applied to the weight matrix to iteratively approximate the solution of the MPF problem.

Let $l_{a \rightarrow P}\left(a_{k, l}, P_{k^{\prime}, l^{\prime}}\right)$ and $l_{a \leftarrow P}\left(a_{k, l}, P_{k^{\prime}, l^{\prime}}\right)$ be the message in the form of log-likelihood ratio (LLR) delivered from $a_{k, l}$ and $P_{k^{\prime}, l^{\prime}}$, respectively. We denote $\xi_{k, l}$ as the set of positions $\left(k^{\prime}, l^{\prime}\right)$ that $a_{k, l}$ interferes on and $\zeta_{k^{\prime}, l^{\prime}}$ as the set of positions $(k, l)$ that interfere on $P_{k^{\prime}, l^{\prime}}$, then

$$
\begin{aligned}
& l_{a \rightarrow P}\left(a_{k, l}, P_{k^{\prime}, l^{\prime}}\right) \\
& =\log \frac{\operatorname{Pr}_{\mathrm{ext}}\left(a_{k, l}=1\right)}{\operatorname{Pr}_{\mathrm{ext}}\left(a_{k, l}=-1\right)} \\
& =\sum_{(n, m) \in \mathcal{\xi}_{k, l} l\left(k^{\prime}, l^{\prime}\right)} l_{a \leftarrow P}\left(a_{k, l}, P_{n, m}\right)
\end{aligned}
$$

By using the message formulated in (14), we have

$$
\operatorname{Pr}_{\mathrm{ext}, k^{\prime}, l^{\prime}}\left(a_{k, l}\right)=\lambda_{k, l} \exp \left(\frac{a_{k, l}}{2} l_{a \rightarrow P}\left(a_{k, l}, P_{k^{\prime}, l^{\prime}}\right)\right)
$$

where $\lambda_{k, l}$ is chosen such that $\operatorname{Pr}_{\mathrm{ext}, k^{\prime}, l^{\prime}}\left(a_{k, l}=+1\right)+\operatorname{Pr}_{\mathrm{ext}, k^{\prime}, l^{\prime}}\left(a_{k, l}=-1\right)=1$, and the subscript "ext" denotes that only the extrinsic information is used.

The inference of parity symbol $P_{k^{\prime}, l^{\prime}}$ to data symbol $a_{k, l}$ is updated as follows

$$
\begin{aligned}
& l_{a \leftarrow P}\left(a_{k, l}, P_{k^{\prime}, l^{\prime}}\right) \\
& =\log \frac{p_{\mathrm{ext}, k^{\prime}, l^{\prime}}\left(a_{k, l}=+1 \mid P_{k^{\prime}, l^{\prime}}, \boldsymbol{a}^{\left[k^{\prime}, l^{\prime}\right]} \backslash a_{k, l}\right)}{p_{\mathrm{ext}, k^{\prime}, l^{\prime}}\left(a_{k, l}=-1 \mid P_{k^{\prime}, l^{\prime}}, \boldsymbol{a}^{\left[k^{\prime}, l^{\prime}\right]} \backslash a_{k, l}\right)} \\
& =\log \frac{p_{\mathrm{ext}, k^{\prime}, l^{\prime}}\left(P_{k, l} \mid \boldsymbol{a}^{\left[k^{\prime}, l^{\prime}\right]}, a_{k, l}=+1\right) \operatorname{Pr}\left(\boldsymbol{a}^{\left[k^{\prime}, l^{\prime}\right]} \backslash a_{k, l}\right)}{p_{\mathrm{ext}, k^{\prime}, l^{\prime}}\left(P_{k, l} \mid \boldsymbol{a}^{\left[k^{\prime}, l^{\prime}\right]}, a_{k, l}=-1\right) \operatorname{Pr}\left(\boldsymbol{a}^{\left[k^{\prime}, l^{\prime}\right]} \backslash a_{k, l}\right)}
\end{aligned}
$$

where the second equality holds following the Bayes rule of (8). Based on MPA, the a priori PMF of $a_{k, l}$ is not included in the computation of a posteriori PMF of $a_{k, l}$.

Substituting (13)-(15) into (16), we can derive $l_{a \leftarrow P}\left(a_{k, l}, P_{k^{\prime}, l^{\prime}}\right)$ in the expression of (17), where $\backslash$ in (16) represents exception.

$$
l_{a \leftarrow P}\left(a_{k, l}, P_{k^{\prime}, l^{\prime}}\right)=\log \frac{\sum_{\boldsymbol{a}^{\left[k^{\prime}, l^{\prime}\right]} \in \mathbb{X}} \exp \left(\sum_{(n, m) \in \epsilon_{k^{\prime}, l^{\prime}} \backslash(k, l)} \frac{a_{n, m} m}{2} l_{a \rightarrow P}\left(a_{n, m}, P_{k^{\prime}, l^{\prime}}\right)-\frac{1}{2 \sigma^{2}}\left\|P_{k^{\prime}, l^{\prime}}-a^{\left[k^{\prime}, l^{\prime}\right]} \boldsymbol{s}^{T}\right\|^{2}\right)}{\sum_{\substack{\boldsymbol{a}^{\left.\prime k^{\prime}, l^{\prime}\right]} \in \mathbb{X} \\ a_{k, l}=-1}} \exp \left(\sum_{(n, m) \in \epsilon_{k^{\prime}, l^{\prime}} \backslash(k, l)} \frac{a_{n, m}}{2} l_{a \rightarrow P}\left(a_{n, m}, P_{k^{\prime}, l^{\prime}}\right)-\frac{1}{2 \sigma^{2}}\left\|P_{k^{\prime}, l^{\prime}}-a^{\left[k^{\prime}, l^{\prime}\right]} s^{T}\right\|^{2}\right)}
$$


When LLR is converged or the maximum number of iterations is reached, the final estimated inference of $a_{k, l}$ will be calculated as

$$
l_{k, l}\left(a_{k, l}\right)=\sum_{(n, m) \in \mathcal{\zeta}_{k, l}} l_{a \leftarrow P}\left(a_{k, l}, P_{n, m}\right)
$$

Consequently, the hard decision of $a_{k, l}$ is

$$
\hat{a}_{k, l}=\arg \max _{a_{k^{\prime}, l^{\prime}} \in \mathbb{X}} l_{k^{\prime}, l^{\prime}}\left(a_{k, l}\right)
$$

\section{Evaluation of IIU}

In this section, we evaluate the proposed IIU to show its effectiveness. The Monte Carlo simulations are conducted over a multipath fading channel of type SUI-3 with 3 paths [22]. The FFT size is 64 , the block length is 16 , the modulation is 4-OQAM and the maximum number of iterations of IIU is 6.

\subsection{Performance with Perfect Channel Estimation and Synchronization}

Figure 1 shows BER comparisons between conventional FBMC-IOTA and our proposed scheme. Please note that in the simulations, perfect channel estimation and synchronization are assumed. For uncoded systems (without channel coding), the intrinsic interference use technique demonstrates about $1 \mathrm{~dB}$ gain over conventional FBMC-IOTA. Moreover, the performance comparison is also provided when a $(1024,512)$ quasi-cyclic LDPC code (LDPC1 coded in the figure) and a $(4096,2048)$ irregular repeat accumulate code (LDPC2 coded in the figure) are adopted to the systems. It can be seen that when different LDPC codes are used, the intrinsic interference use still achieves noticeable performance improvement.

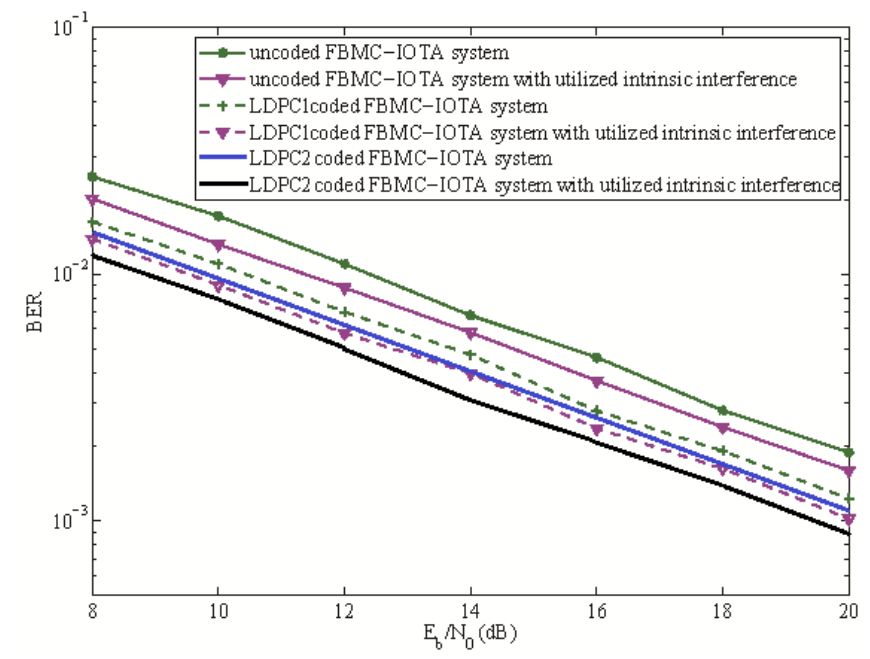

Figure 1. BER performance with perfect channel estimation and synchronization.

\subsection{Effect of Carrier Frequency Offset (CFO)}

The received signal (after equalizing the channel effect) in the presence of CFO becomes

$$
r(t)=s(t) \exp \left(2 \pi j f_{0} t\right)
$$

Hence the desired symbol $a_{k, l}$ is demonstrated as 


$$
\begin{aligned}
& \hat{a}_{k, l} \\
& =\int r(t) g_{k, l}^{*}(t) d t \\
& =\int s(t) \exp \left(2 \pi j f_{0} t\right) g_{k, l}^{*}(t) d t \\
& =\int \sum_{n, m} a_{n, m} g_{n, m}(t) g_{k, l}^{*}(t) \exp \left(j 2 \pi f_{0} t\right) d t \\
& =\sum_{n, m} a_{n, m} \int g\left(t-n \tau_{0}\right) g^{*}\left(t-l \tau_{0}\right) \exp \left(2 \pi j f_{0} t\right) \\
& \exp (j(m+n-k-l) \pi / 2) \exp \left(2 j \pi(m-k) v_{0} t\right) d t
\end{aligned}
$$

Denoting $t=x+\frac{(n+l) \tau_{0}}{2}, \hat{a}_{k, l}$ can be calculated by (22). Moreover, considering that $f_{0}=\delta v_{0},(22)$ becomes (23) which shows that a frequency offset will change the weights and some of the intrinsic interference will be added to the desired signal. Under this condition, new weights should be calculated. Figure 2 shows how the FBMC-IOTA system with $15 \mathrm{kHz}$ subcarrier spacing are affected by frequency offset of $60 \mathrm{~Hz}$. As indicated by the figure, the gain obtained by IIU reduces due to the frequency offset. This follows from the fact that frequency offset will distort the desired signal and subsequently affects the calculation of parity symbols in the algorithm.

$$
\begin{aligned}
& \hat{a}_{k, l}=\sum_{n, m} a_{n, m} \int g\left(x+\frac{l-n}{2} \tau_{0}\right) g^{*}\left(x-\frac{l-n}{2} \tau_{0}\right) \\
& \exp (j(m+n-k-l+(m-k)(n+l)) \pi / 2) \exp \left(2 \pi j\left((m-k) v_{0}+f_{0}\right) x\right) \exp \left(2 \pi j f_{0} \frac{n+l}{2} \tau_{0}\right) d x \\
& =\sum_{n, m} a_{n, m}(j)^{m+n-k-l+(m-k)(n+l)} \exp \left(2 \pi j f_{0} \frac{n+l}{2} \tau_{0}\right) A_{g}\left((l-n) \tau_{0},(m-k) v_{0}+f_{0}\right) \\
& \hat{a}_{k, l}=\sum_{n, m} a_{n, m}(j)^{m+n-k-l+(m-k)(n+l)} \exp \left(\pi j \delta \frac{n+l}{2}\right) A_{g}\left((l-n) \tau_{0},(m-k+\delta) v_{0}\right)
\end{aligned}
$$

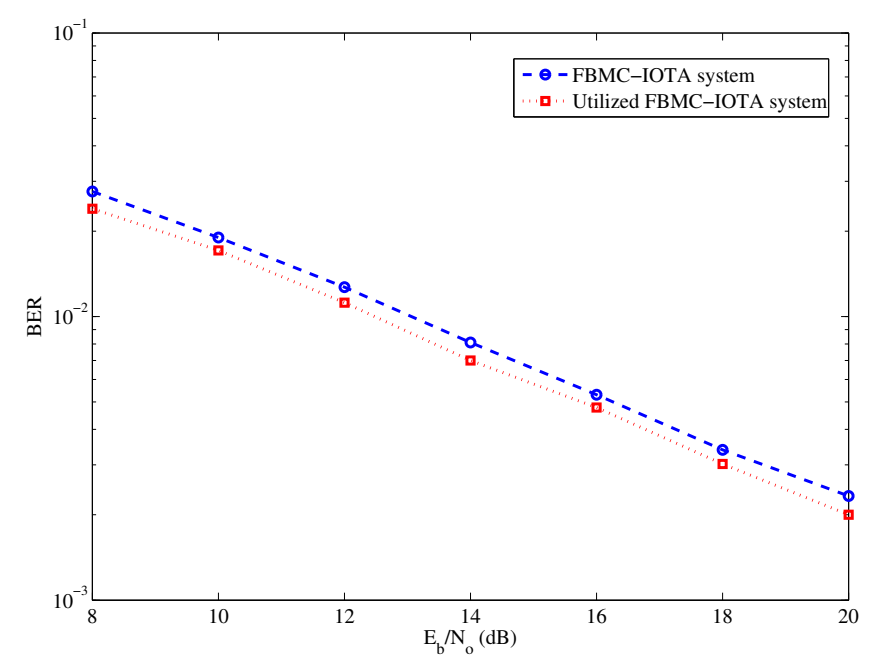

Figure 2. Effect of CFO.

\subsection{Effect of Imperfect Channel Estimation}

Conventional channel estimation methods used for OFDM cannot be directly applied to FBMC-IOTA due to the intrinsic interference. To address this problem several preamble-based algorithms have been proposed in the literature [15]. We consider that the estimated channel is 
correlated with the true channel with a correlation factor $\rho_{e}=E\left[\hat{H}_{k, l}, H_{k, l}\right]$, where $H_{k, l}$ represents the channel coefficient at the $l$ th symbol and the $k$ th subcarrier frequency after the IOTA filtering and FFT operation. The estimated channel can be modeled as

$$
\hat{H}_{m, n}=\rho_{e} H_{m, n}+\sqrt{1-\rho_{e}^{2}} E_{m, n}
$$

where $E_{m, n}$ represents the Gaussian error signal and independent of the real channel. Figure 3 shows that imperfect channel estimation will change parity symbols, consequently the IIU algorithm and its gain are slightly affected. Any discrepancy in the performance is due to the fundamental dependence of the schemes on channel state information (CSI), rather than the particular channel estimation procedures employed. In future we aim to study a scattered pilot-based channel estimation scheme which benefits from the intrinsic interference calculations we carried out in this paper.

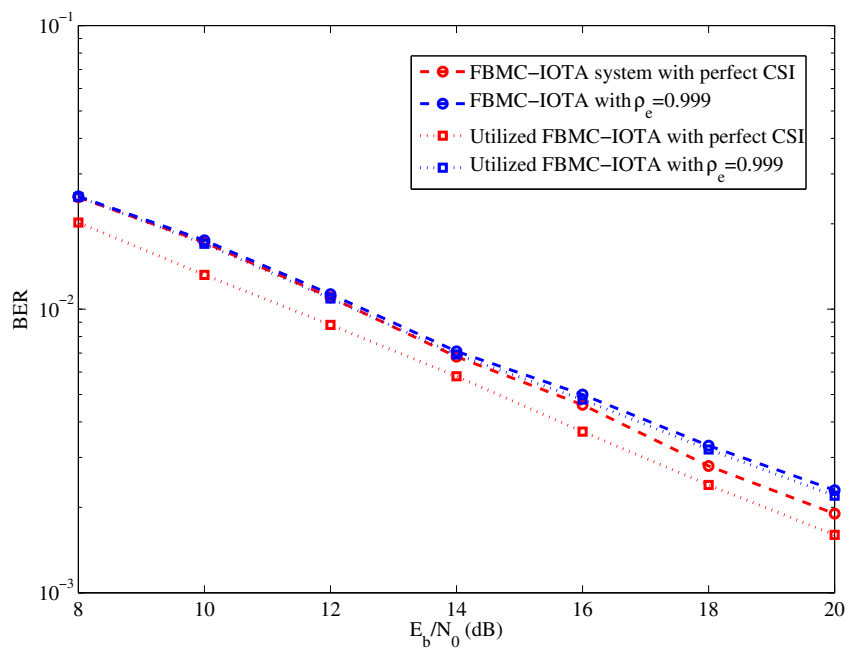

Figure 3. Effect of imperfect channel estimation.

\subsection{Complexity of Proposed Algorithm}

As explained earlier, we reduced the complexity of the IIU by eliminating some low valued weights. Let us define $d_{c}$ as the effective number of weights, which corresponds to the effective number of neighboring symbols that interfere with each other on each parity symbol. Denote $D$ as the total number of neighboring symbols (including those with low valued weights) that interfere on each parity symbol. In the proposed algorithm at each received parity symbol, each symbol has only $d_{c}-1$ interferers, where $d_{c}<D$. The complexity order of the proposed IIU algorithm is $\mathcal{O}\left(|\mathbb{X}|^{d_{c}}\right)$, which is much less than $\mathcal{O}\left(|\mathbb{X}|^{D}\right)$ (the optimal case). The complexity of IIU for FBMC-IOTA is shown in (25).

$$
\text { Complexity }=|\mathbb{X}|^{d_{c}} \times(\text { Iteration number }) \times(\text { No. of parity symbols in error })
$$

Please note that the highest possible value for the number of erroneous parity symbols is $N \times L$. Apparently, there is a tradeoff between the accuracy of the algorithm and the complexity. Higher values of $d_{c}$ will result in more accurate detection but the complexity will increase exponentially with regards to $d_{c}$. We set $d_{c}=6$, as it is an appropriate value to strike the balance between the accuracy of the algorithm and the complexity.

\section{Conclusions}

A novel algorithm for FBMC-IOTA systems with IOTA pulse-shaping is proposed to use the intrinsic interference in the demodulation process. The intrinsic interference is treated as parity symbols, which enables us to correct errors using the information offered by these parity symbols. 
Based on MPA, IIU is developed to improve the system performance. Effects of CFO and imperfect channel estimation on IIU are investigated.

Author Contributions: Conceptualization, L.W. and R.R.; methodology, J.L.; software, J.L.; validation, R.R. and J.L.; formal analysis, L.W.; investigation, R.R.; resources, J.L.; data curation, R.R.; writing-original draft preparation, J.L.; writing-review and editing, L.W.; visualization, R.R.; supervision, J.L.; project administration, L.W.; funding acquisition, L.W.

Funding: This work was supported in part by the National Defense Technology Foundation under Grant 3101168, in part by the Hunan Natural Science Foundation under Grant 2017JJ2303, and in part by National Natural Science Foundation of China under Grant 61603007 and Grant 61702536.

Conflicts of Interest: The authors declare no conflict of interest.

\section{References}

1. Banelli, P.; Buzzi, S.; Colavolpe, G.; Modenini, A.; Rusek, F.; Ugolini, A. Modulation formats and waveforms for 5G networks: Who will be the heir of OFDM: An overview of alternative modulation schemes for improved spectral efficiency. IEEE Signal Process. Mag. 2014, 31, 80-93. [CrossRef]

2. Wen, L.; Razavi, R.; Imran, M.A.; Xiao, P. Design of joint sparse graph for OFDM system. IEEE Trans. Wirel. Commun. 2014, 14, 1823-1836. [CrossRef]

3. Nam, H.; Choi, M.; Han, S.; Kim, C.; Choi, S.; Hong, D. A new filter-bank multicarrier system with two prototype filters for QAM symbols transmission and reception. IEEE Trans. Wirel. Commun. 2016, 15, 5998-6009. [CrossRef]

4. Saeedi-Sourck, H.; Yan, W.; Bergmans, J.W.M. Complexity and performance comparison of filter bank multicarrier and OFDM in uplink of multicarrier multiple access networks. IEEE Trans. Signal Process. 2011, 59, 1907-1912. [CrossRef]

5. Lopez-Salcedo, J.A.; Gutierrez, E.; Seco-Granados, G.; Swindlehurst, A.L. Unified framework for the synchronization of flexible multicarrier communication signals. IEEE Trans. Signal Process. 2013, 61, 828-842. [CrossRef]

6. Cui, W.; Qu, D.; Jiang, T.; Farhang-Boroujeny, B. Coded auxiliary pilots for channel estimation in FBMC-OQAM systems. IEEE Trans. Veh. Technol. 2016, 65, 2936-2946. [CrossRef]

7. Verde, F.; Gelli, G.; Paura, L.; Darsena, D. Widely-linear equalisation and blind channel identification for interference-contaminated multicarrier systems. IEEE Trans. Signal Process. 2004, 51, 1153-1164.

8. Darsena, D.; Verde, F. Minimum-mean-output-energy blind adaptive channel shortening for multicarrier SIMO transceivers. IEEE Trans. Signal Process. 2007, 19, 119-134. [CrossRef]

9. Darsena, D.; Gelli, G.; Verde, F. Universal linear precoding for NBI-proof widely-linear equalization in MC systems. EURASIP J. Wirel. Commun. 2008, 19, 1634-1641. [CrossRef]

10. Darsena, D.; Gelli, G.; Paura, L. A constrained maximum-SINR NBI-resistant receiver for OFDM systems. IEEE Trans. Signal Process. 2007, 43, 3032-3047. [CrossRef]

11. Bolcskei, H.; Duhamel, P.; Hleiss, R. Design of pulse shaping OFDM/OQAM systems for high data-rate transmission over wireless channels. In Proceedings of the IEEE International Conference on Communications ICC '99, Vancouver, BC, Canada, 6-10 June 1999; Volume 1, pp. 559-564.

12. Vangelista, L.; Laurenti, N. Efficient implementations and alternative architectures for OFDM-OQAM systems. IEEE Trans. Commun. 2001, 49, 664-675. [CrossRef]

13. Liu, Y.; Chandrasekaran, V.; Anandkumar, A. Feedback message passing for inference in Gaussian graphical models. IEEE Trans. Signal Process. 2012, 60, 4135-4150. [CrossRef]

14. Farhang, B. OFDM Versus Filter Bank Multicarrier. IEEE Signal Process. Mag. 2011, 28, 92-112. [CrossRef]

15. Du, J.; Signell, S. Novel Preamble-Based Channel Estimation for OFDM/OQAM Systems. In Proceedings of the 2009 IEEE International Conference on Communications (ICC), Dresden, Germany, 14-18 June 2009.

16. Razavi, R.; Xiao, P.; Tafazolli, R. Information theoretic analysis of OFDM/OQAM with utilized intrinsic interference. IEEE Signal Process. Lett. 2016, 22, 618-622. [CrossRef]

17. Lee, S.H.; Shamaiah, M.; Vikalo, H.; Vishwanath, S. Message-Passing Algorithms for Coordinated Spectrum Sensing in Cognitive Radio Networks. IEEE Commun. Lett. 2013, 17, 812-815.

18. Verdu, S. Multiuser Detection; Cambridge University Press: Cambridge, UK, 1998. 
19. Poon, T.V.; Beaulieu, N.C. Jointly and individually optimum receivers for BPSK signals in cochannel interference plus noise. In Proceedings of the 2003 IEEE Pacific Rim Conference on Communications Computers and Signal Processing (PACRIM 2003) (Cat. No. 03CH37490), Victoria, BC, Canada, 28-30 August 2003; Volume 2, pp. 530-532.

20. Lupas, R.; Verdu, S. Linear multiuser detectors for synchronous code-division multiple-access channels. IEEE Trans. Inf. Theory 1989, 35, 123-136. [CrossRef]

21. Kschischang, F.; Frey, B. Iterative decoding of compound codes by probability propagation in graphical models. IEEE J. Sel. Areas Commun. 1998, 16, 219-230. [CrossRef]

22. Erceg, V.; Greenstein, L.; Tjandra, S.; Parkoff, S.; Gupta, A.; Kulic, B.; Julius, A.; Bianchi, R. An empirically based path loss model for wireless channels in suburban environments. IEEE J. Sel. Areas Commun. 1999, 17, 1205-1211. [CrossRef]

(C) 2019 by the authors. Licensee MDPI, Basel, Switzerland. This article is an open access article distributed under the terms and conditions of the Creative Commons Attribution (CC BY) license (http://creativecommons.org/licenses/by/4.0/). 